\title{
The Effect of Group Counseling on the Quality of Life in Patients with Major Thalassemia Referred to the Thalassemia Treatment Center in Bushehr
}

\author{
Rezvan Bakhshi (MSc) ${ }^{1}$, Sherafat Akaberian (MSc) $)^{1, *}$, Masoud Bahreini (PhD) ${ }^{1}$, \\ Kamran Mirzaei $(\mathbf{P h D})^{2}$, Jamileh Kiani (MSc $)^{3}$ \\ ${ }^{I}$ Nursing Department, Faculty of Nursing and Midwifery, Bushehr University of Medical Science, Bushehr, Iran \\ ${ }^{2}$ Social Medicine Department, Faculty of Medicine, Bushehr University of Medical Science, Bushehr, Iran \\ ${ }^{3}$ Bushehr University of Medical Science, Bushehr, Iran
}

* Corresponding Author: Sherafat Akaberian, Nursing Department, Faculty of Medicine, Bushehr University of Medical Science, Bushehr, Iran.Email: sh.akabarian@bpums.ac.ir

\begin{tabular}{|c|c|}
\hline \multirow[b]{2}{*}{$\begin{array}{l}\text { Received: } 12 / 04 / 2017 \\
\text { Accepted: } 21 / 03 / 2018\end{array}$} & Abstract \\
\hline & \multirow{8}{*}{$\begin{array}{l}\text { Background and Objective: Major Thalassemia is the most common } \\
\text { hereditary disease in the world and in Iran. The chronic nature of the } \\
\text { disease and complications associated with clinical signs and protests of the } \\
\text { disease and its treatment make multiple physical, psychological and social } \\
\text { problems and effects on the quality of life in these patients. The aim of this } \\
\text { study was to determine the effect of group counseling on quality of life in } \\
\text { patients with thalassemia major. } \\
\text { Materials and Methods: This study was a randomized controlled clinical } \\
\text { trial. The total number of subjects included } 62 \text { patients with thalassemia } \\
\text { major from thalassemia treatment center in the Bushehr in } 2016 \text {, were } \\
\text { randomly divided into two groups of } 31 \text { people of intervention and control. } \\
\text { For intervention group, four sessions of group counseling in behavioral } \\
\text { cognitive approach held in two weeks. The quality of life in patients in both } \\
\text { groups evaluated before the intervention and one month after the } \\
\text { intervention, using the SF-36 questionnaire. The results were analyzed by } \\
\text { SPSS software version } 23 \text { at a significant level of } 0.05 \text {. } \\
\text { Results: The results of analytical tests showed that there was no } \\
\text { statistically significant difference between the two groups in demographic } \\
\text { variables (P > 0.05). However, the intervention group gained a significant } \\
\text { increase in total score of quality of life and its sub-domains after } \\
\text { counseling. (P }<0 / 001) \text {. Also, the difference between the mean total scores } \\
\text { of quality of life and its sub-domains in the intervention and control groups } \\
\text { was statistically significant }(\mathrm{P}<0.001) \text {. } \\
\text { Conclusion: This study showed that the treatment group counseling with } \\
\text { cognitive behavioral approach significantly improves the quality of life in } \\
\text { patients with Thalassemia major. Therefore it is recommended that this } \\
\text { treatment helped to improve the quality of life in these patients. }\end{array}$} \\
\hline \multirow{8}{*}{$\begin{array}{l}\text { How to Cite this Article: } \\
\text { Bakhshi R, Akaberian Sh, } \\
\text { Bahreini M, Mirzaei K, Kiani J. } \\
\text { The Effect of Group Counseling } \\
\text { on the Quality of Life in Patients } \\
\text { with Major Thalassemia } \\
\text { Referred to the Thalassemia } \\
\text { Treatment Center in Bushehr. } \\
\text { Pajouhan Scientific Journal. } \\
\text { 2018; 16(3): 11-19. DOI: } \\
\text { 10.18869/psj.16.3.11 }\end{array}$} & \\
\hline & \\
\hline & \\
\hline & \\
\hline & \\
\hline & \\
\hline & \\
\hline & $\begin{array}{l}\text { Keywords: Beta Thalassemia; Cognitive-Behavioral Approach; Counseling; } \\
\text { Quality of Life }\end{array}$ \\
\hline
\end{tabular}

Copyright () 2018 Pajouhan Scientific Journal. This is an open-access article distributed under the terms of the Creative Commons Attribution-NonCommercial 4.0 International License (http://creativecommons.org/licenses/by-nc/4.0/) which permits copy and redistribute the material just in noncommercial usages, provided the original work is properly cite. 
do): $10.18869 /$ psj. 16.3 .11

بررسى تاثير مشاوره گروهى بر كيفيت زندىى بيماران مبتلا به تالاسـمى مـازور مراجعـــ كننله به مركز درمانى تالاسمى بوشهر

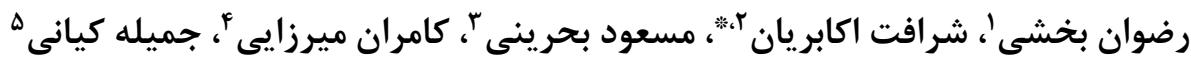 \\ ' دانشجوى كارشناسى ارشد يرستارى كودكان، دانشكده ى يرستارى و مامايى، دانشخاه علوم يزشكى بوشهر، بوشهر، ايران

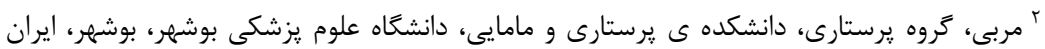

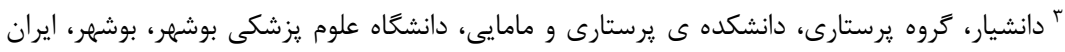

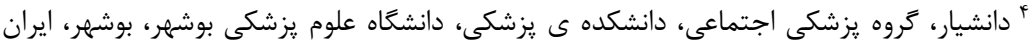

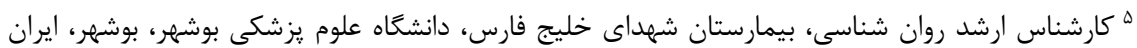

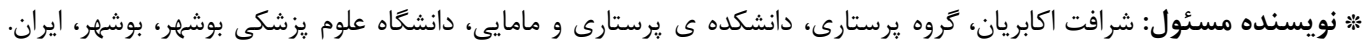 \\ ايميل: sh.akabarian@bums.ac.ir
}

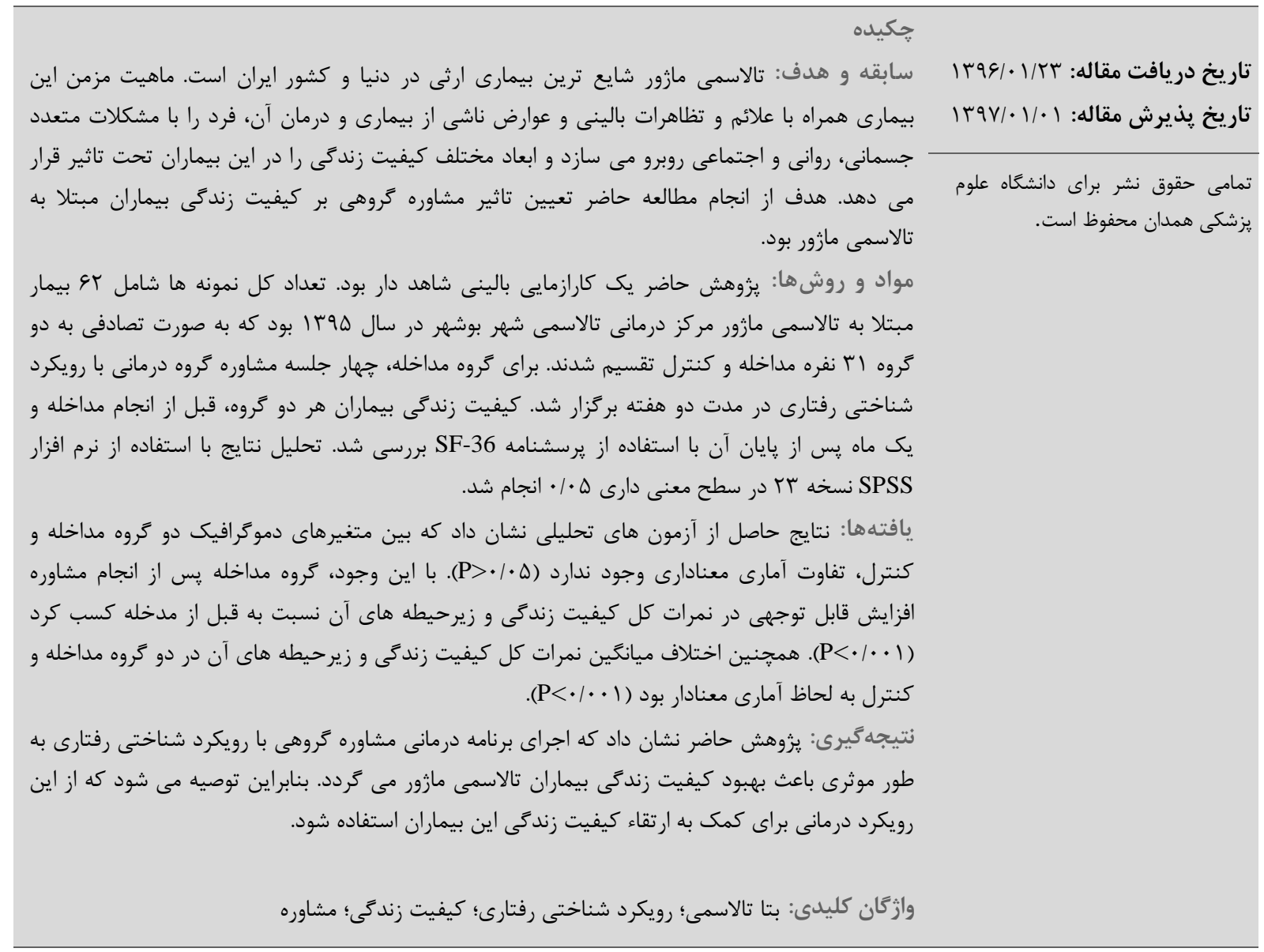

سندرم هاى بتا تالاسمى گروهى از اختلالات وراثتى خونى كئى

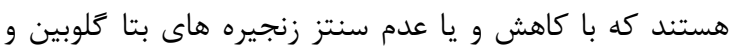

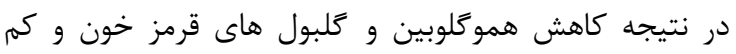

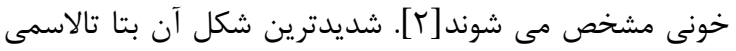

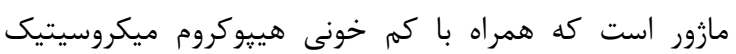

امروزه بيمارى هاى مزمن علت عمده مسائل بهداشتى

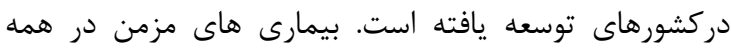

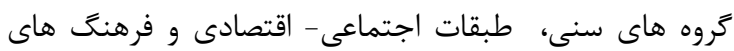
مختلف بروز مى كند. يكى از اين بيمارى هاى مزمن كنه سله سلامت

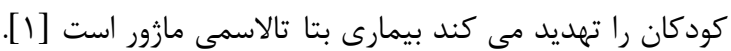


بايد به مهارت هاى زندَّى اين بيماران توجه بيشترى داشته

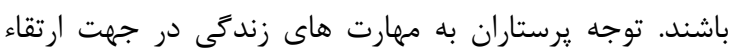

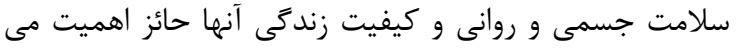

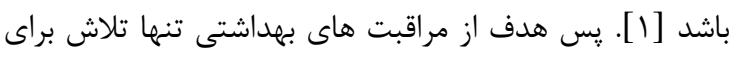

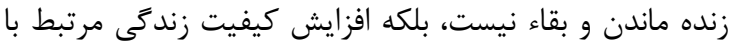

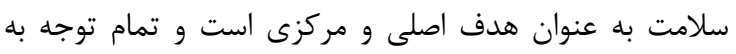

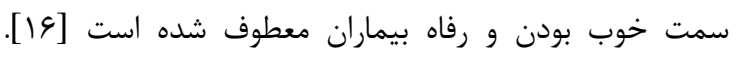

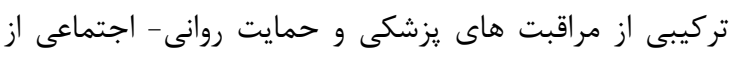

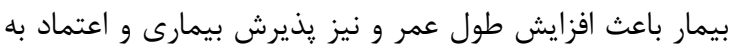

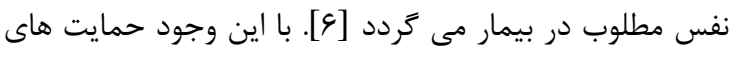

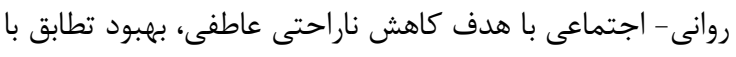

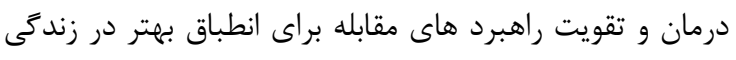

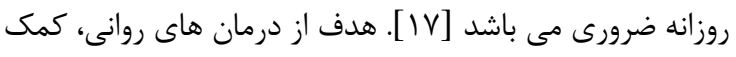

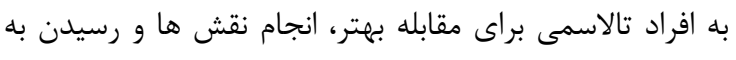

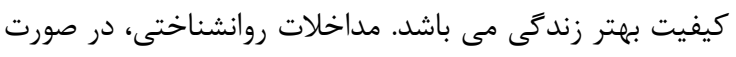

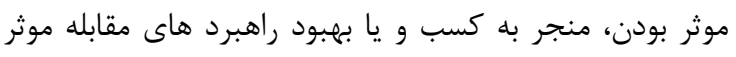

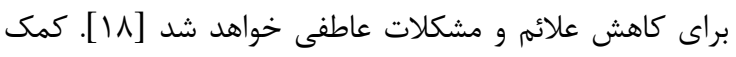

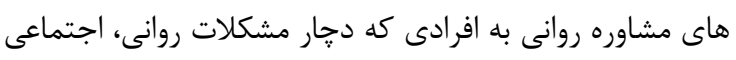

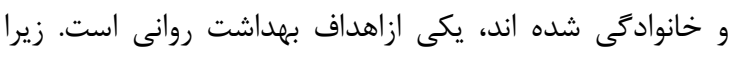

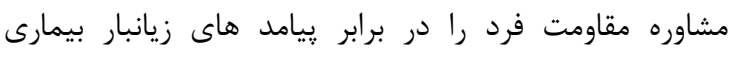

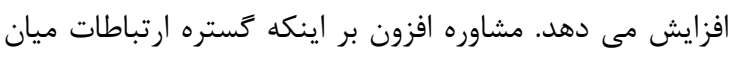

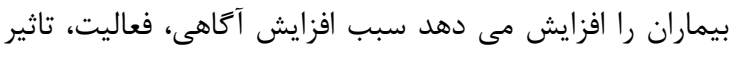

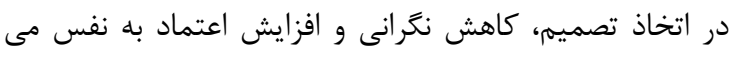

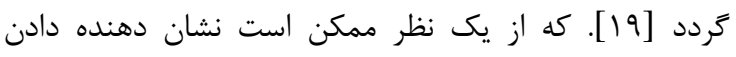

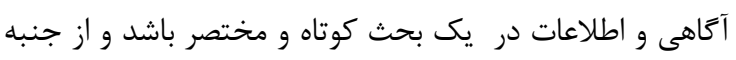

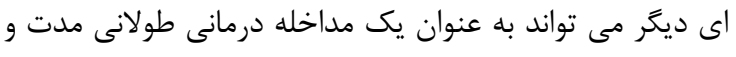

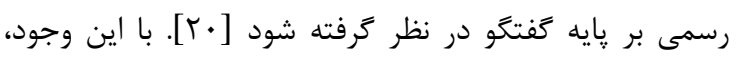

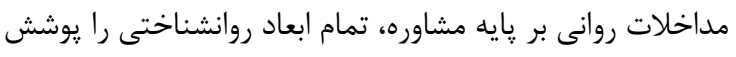

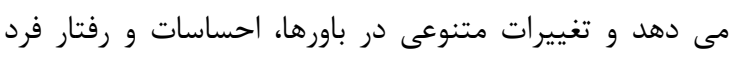

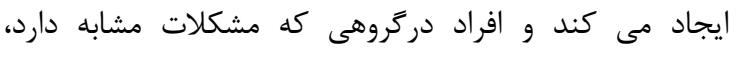

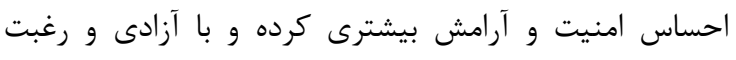

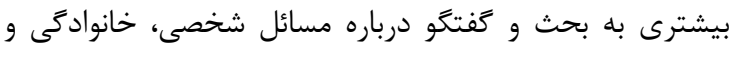

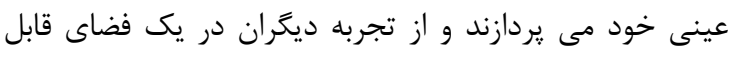

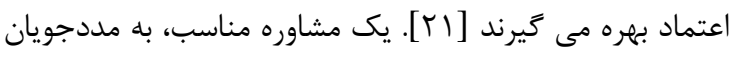

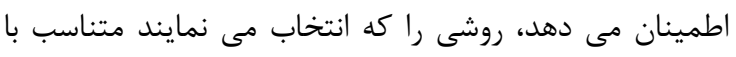

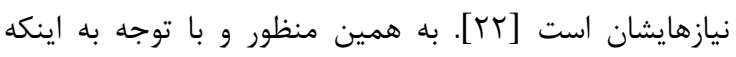

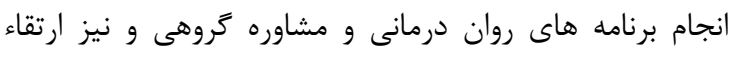

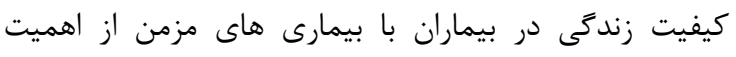

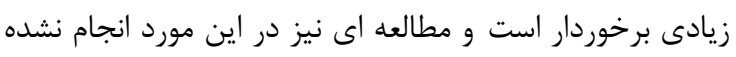

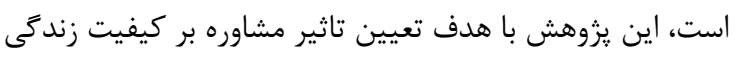
بيماران مبتلا به تالاسمى مازور طراحى ترديد.

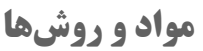

يزوهش حاضر يك كارازمايى بالينى تصادفى شاهد دار با
شديد مى باشد[ب]. سازمان جهانى بهداشت تالاسمى را به

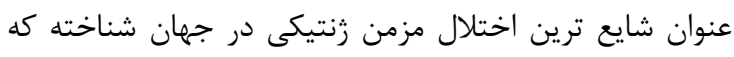

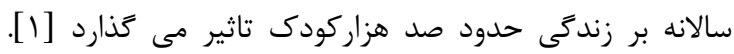

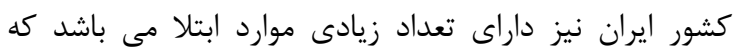

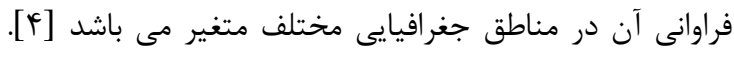

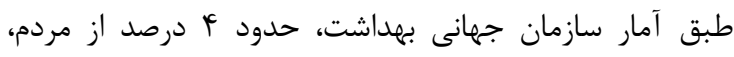

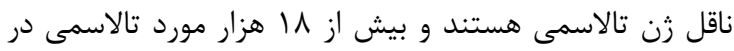

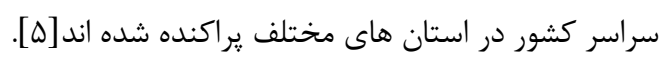

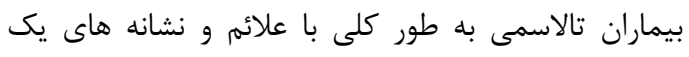

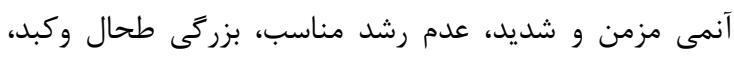

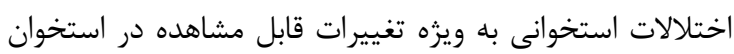

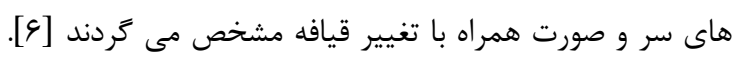

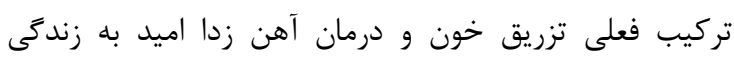

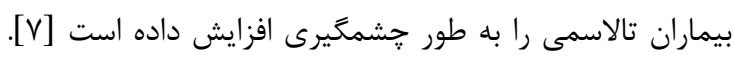

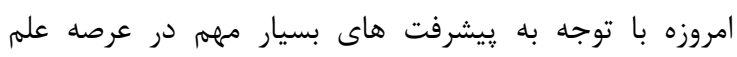

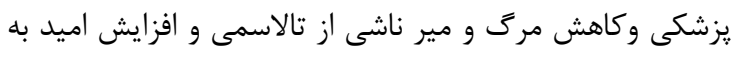

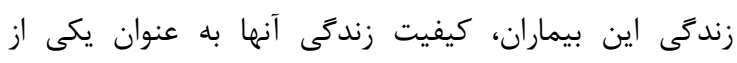

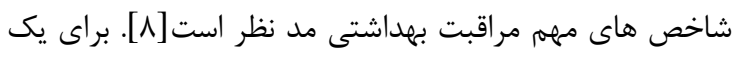

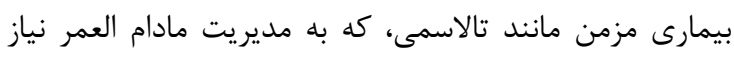

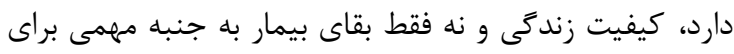

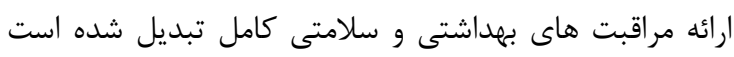

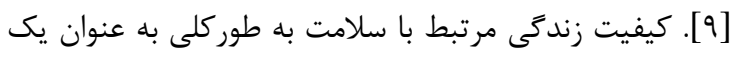

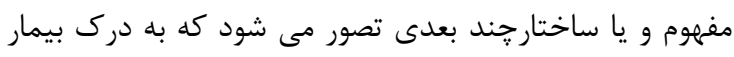

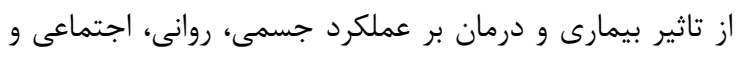

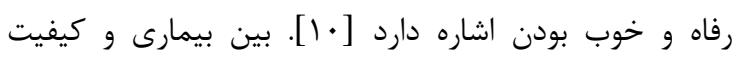

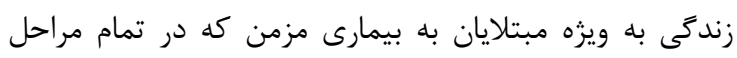

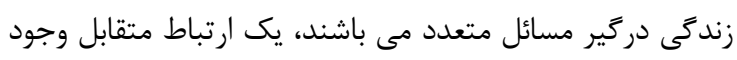

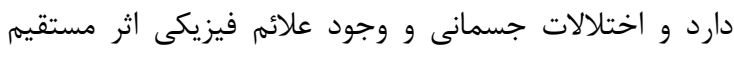

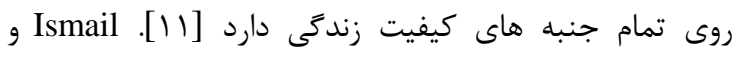

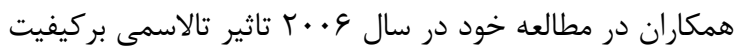

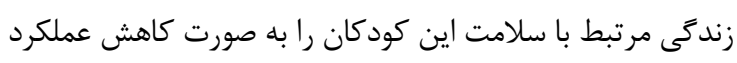

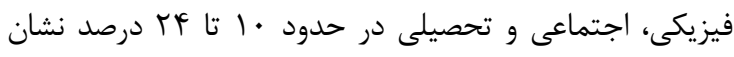

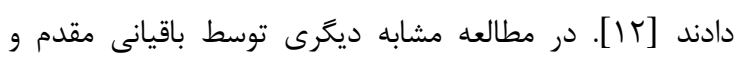

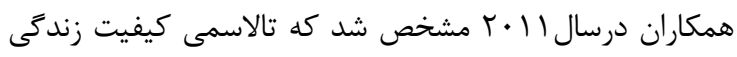

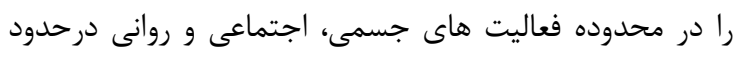

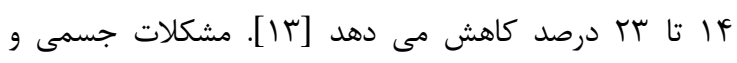

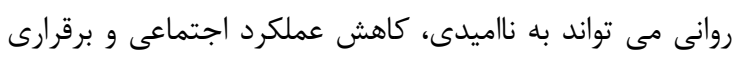

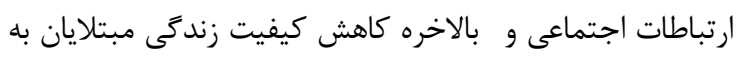

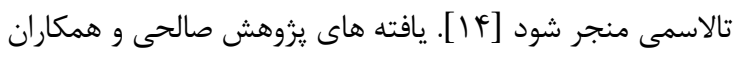

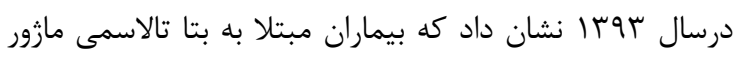

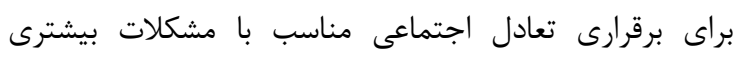

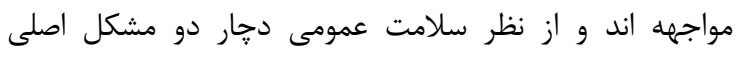

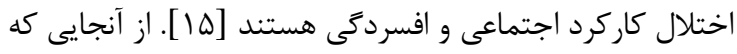

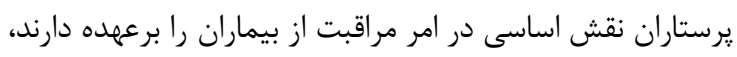


بيش آزمون، ابتدا براساس تعداد بيماران مبتلا به تالاسمى

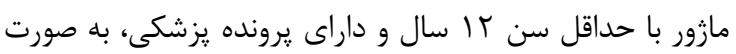
سرشمارى و با توجه به معيارهاى ورود و خروج، سهرم تعداد

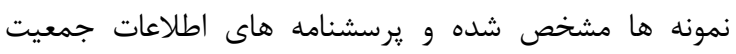
شناختى و كيفيت زندگى SF-36 در اختيار آنها قرار كرفت.

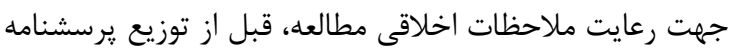

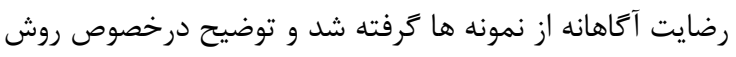

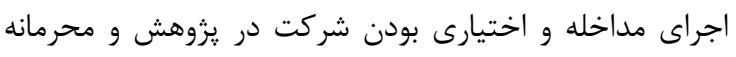

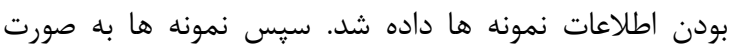

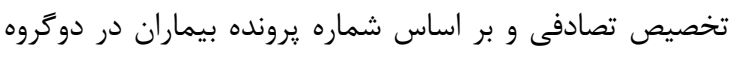

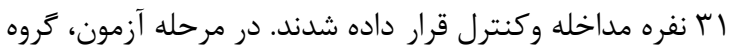

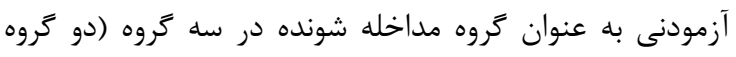

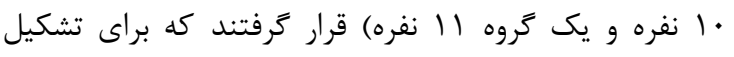
گروه ها و دعوت به شركت در مشاوره با هريك از اعضاى گر زروه

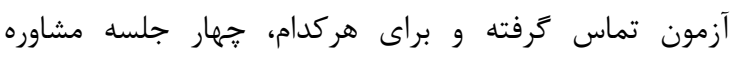

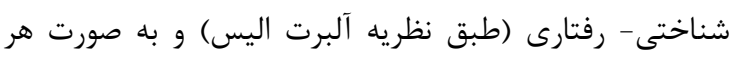

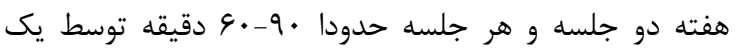

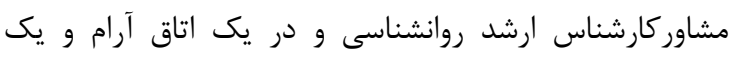

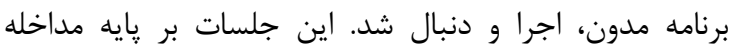

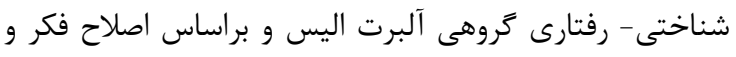

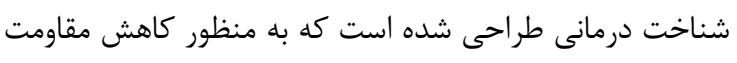

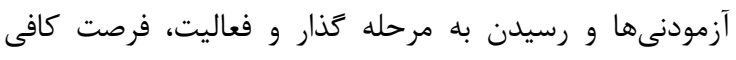

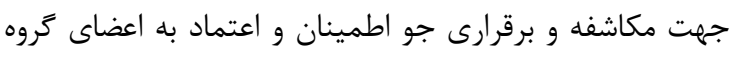

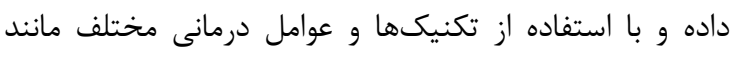
ايفاى نقش، بارش فكرى، معكوس سازى نقش، جانشين سازى

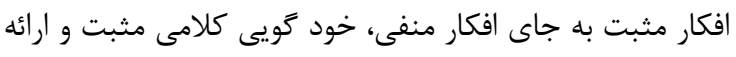

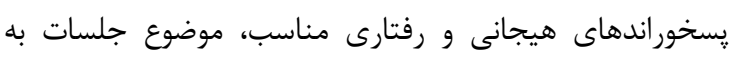
سمت طرح مشكلات مربوط به درمان مادام العمر بيمارى

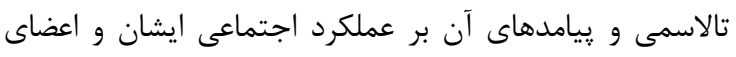

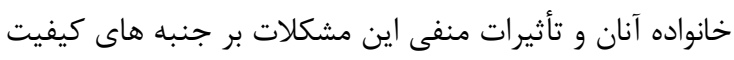

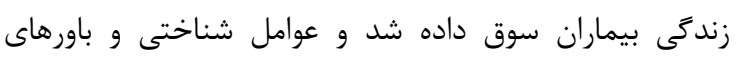

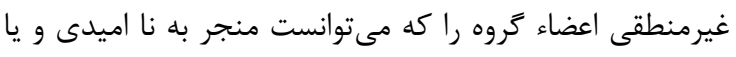

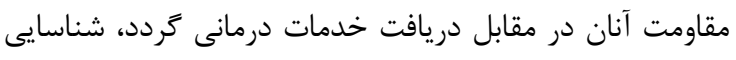

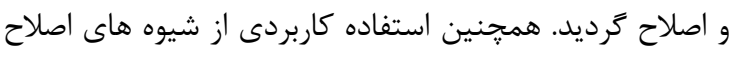

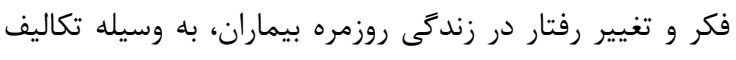

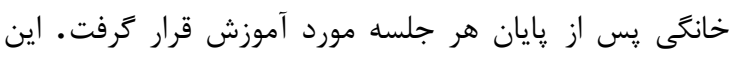

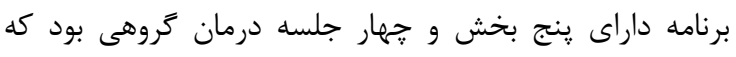

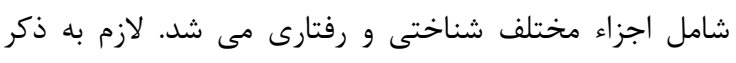

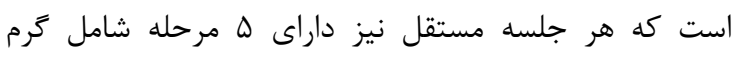

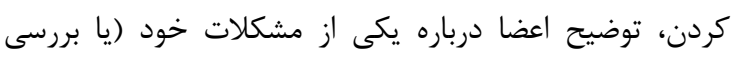

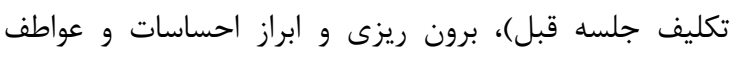

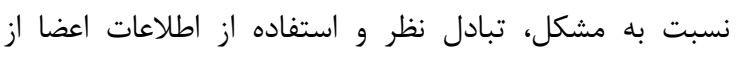
يكديكر، جمع بندى و دادن تكليف جهت جلسه بعد مى شد شد

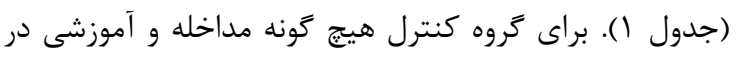

طراحى يِيش آزمون- يس آزمون بود كه در سال هوسا و در

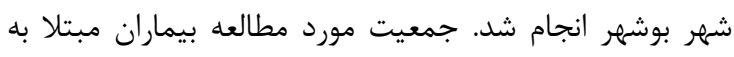

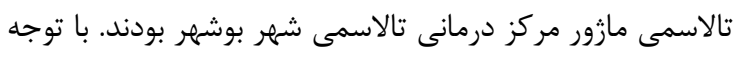
به مطالعه هادى و همكاران [بr] و ميانگين (انحراف معيار)

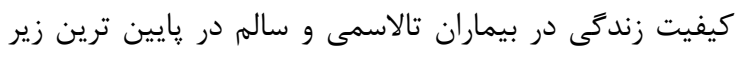

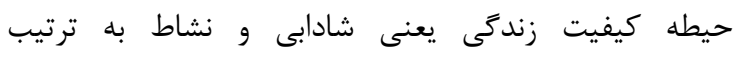

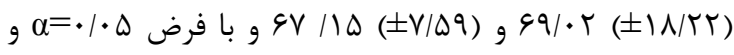

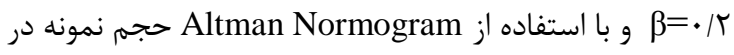
گروه مداخله با احتساب target difference معادل حداقل هץ نفر در هر گروه و در مجموع • V نفر محاسبه كرديد.

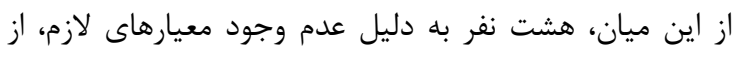

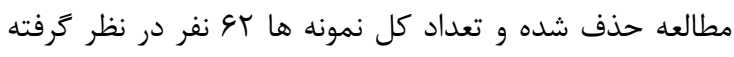

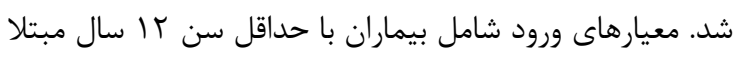

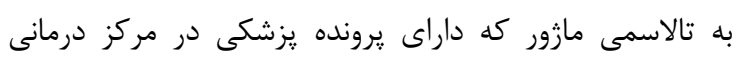

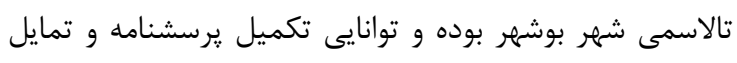

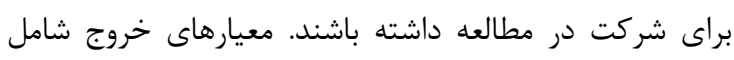

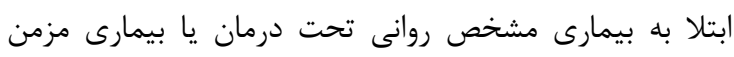

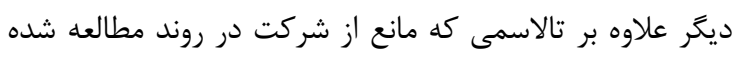

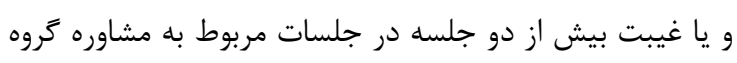

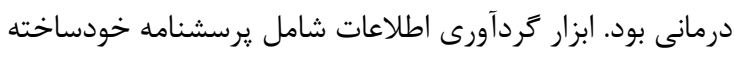

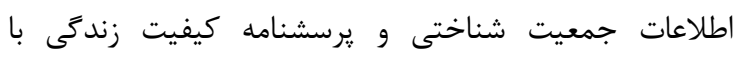

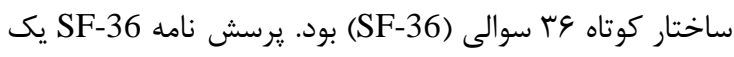

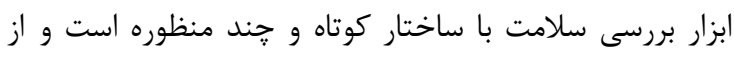

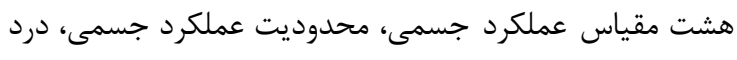

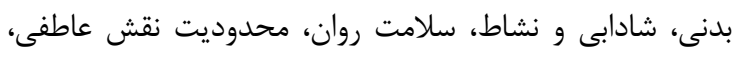

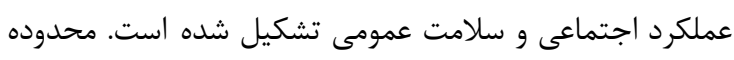

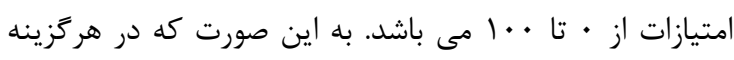

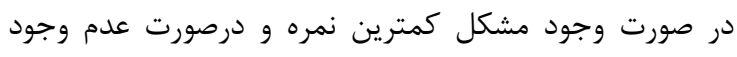

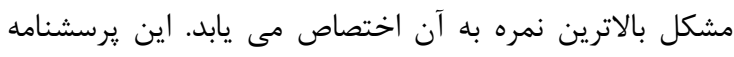

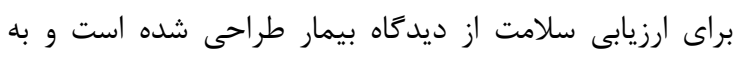

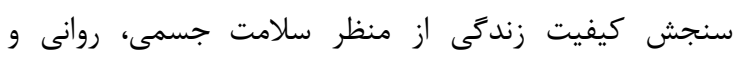

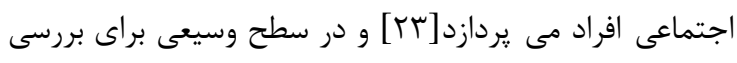

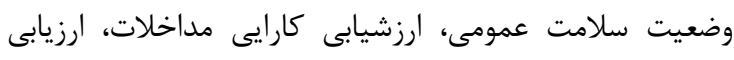

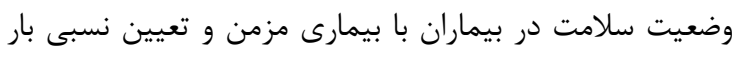

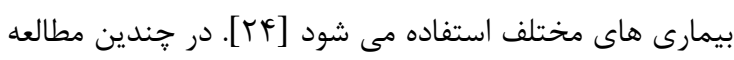

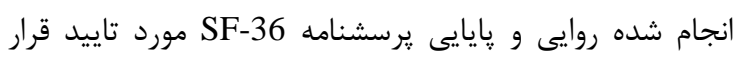

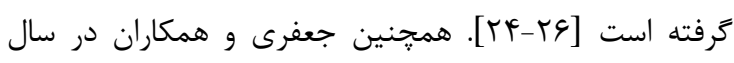

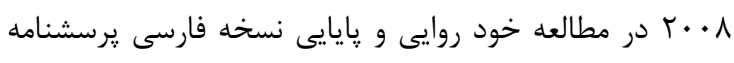

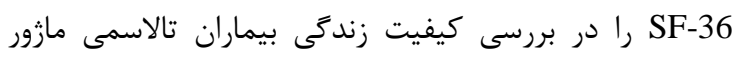

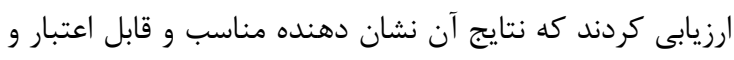

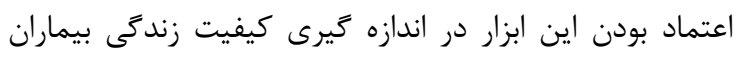

تالاسمى مازور بود [rV]

براى اجراى يزوهش يس مازور بود از تصويب و كسب مجوز از

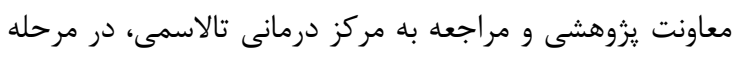


جدول ا: محتواى جلسات گروه درمانى

\begin{tabular}{|c|c|}
\hline محتوا & جلسات \\
\hline شانب اعضاى هدف از تشكيل گروه، جهت گيرى عمومى درمان و بيان نظرات اعضا، توانايى ها و علايق خود و ارائه حمايت از & اول \\
\hline اعضا در رويارويى خود هوشيارى افراد، بررسى مشكلات محيطى و عوامل ناخوشايند در رابطه با بيمارى و تجربيات مثبت و منفى & دوم \\
\hline ايجاد آنى عوامل شناختى در رابطه با رفتار ناسازكارانه و نحوه شكل گيرى افكار مخرب و تجربيات ناخوشايند و دلايل & سوم \\
\hline 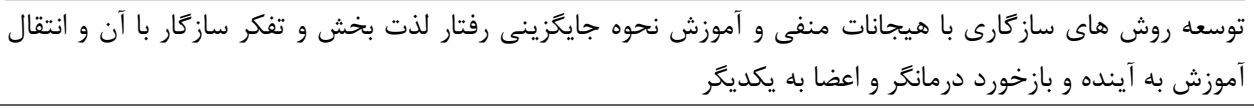 & جهارم \\
\hline
\end{tabular}

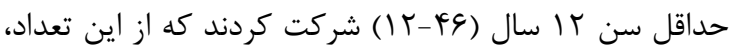

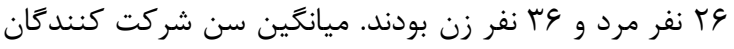

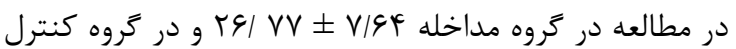
مداخله

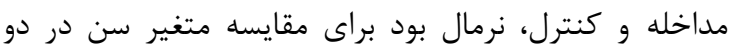

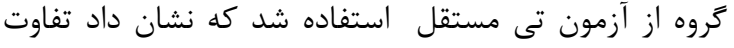

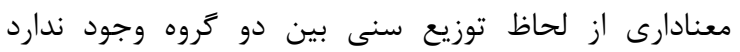

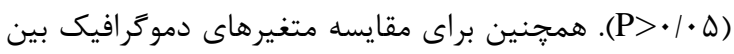

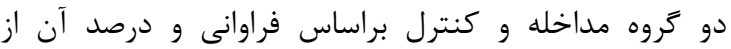

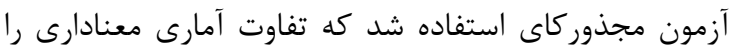

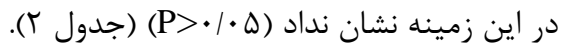

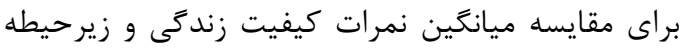
هاى آن قبل و بعد از مداخله در هر دو گروه، از آزمون هاى

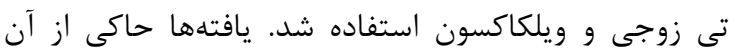

نظر كرفته نشد و تنها تحت مراقبت هاى معمول بخش درمانى

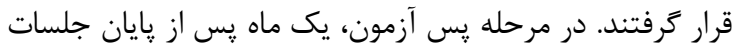

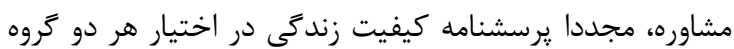

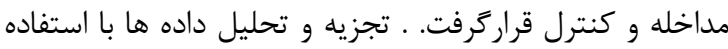
از آماره هاى توصيفى و آزمون مجذور كاى انجام شد. همجني تهنين

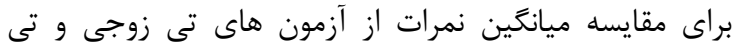

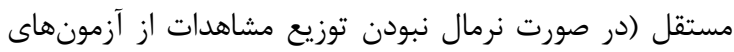

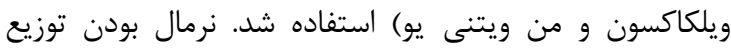

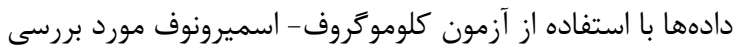

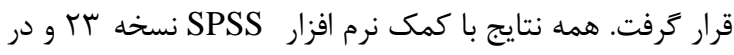
سطح معنى دارى ه • • • بدست آمدند.

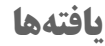

در اين زيزوهش، rو بيمار مبتلا به تالاسمى مازور با

جدول r: فراوانى مشخصات دموَر افيك شركت كنندًان در مطالعه به تفكيك دو گروه مداخله وكنترل

\begin{tabular}{|c|c|c|c|c|}
\hline p-value & 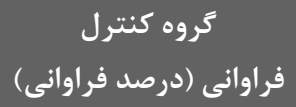 & 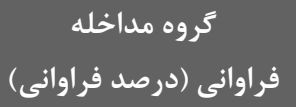 & \multicolumn{2}{|c|}{ اطلاعات دموَر افيك } \\
\hline \multirow{2}{*}{$\cdot 19 \cdot V$} & $1 T(r N / V)$ & $\mid \mathcal{F}(\mathcal{E} \Delta / T)$ & مرد مرد & \multirow[b]{2}{*}{ جنس } \\
\hline & $19(91 / \Gamma)$ & $I V(\Delta F / \Lambda)$ & زن & \\
\hline \multirow{3}{*}{$\cdot / r \wedge V$} & $19(01 / 9)$ & $1 \cdot(r T / T)$ & زير دييلم & \multirow{3}{*}{ نحصيلات } \\
\hline & $V(Y T / G)$ & $\|(r \Delta / \uparrow)$ & دييلمم & \\
\hline & $\wedge(r \Delta / \wedge)$ & $1 \cdot(r r / T)$ & بالاتر از ديڤلم & \\
\hline \multirow{3}{*}{.1991} & $10(F N / F)$ & $\operatorname{Ir}(\mathrm{rN} / \mathrm{V})$ & زير دييلم & \multirow{3}{*}{ تحصيلات ڤدر } \\
\hline & $\Lambda(r \Delta / \Lambda)$ & $11(\Gamma \Delta / \Delta)$ & دييلم & \\
\hline & $\wedge(\Gamma \Delta / \wedge)$ & $\wedge(r \Delta / \wedge)$ & بالاتر از دييلم & \\
\hline \multirow{3}{*}{. MTr } & $r)(\Phi V / V)$ & $1 \wedge(\Delta N / 1)$ & زير دييلهم & \multirow{3}{*}{ تحصيلات مادر } \\
\hline & $V(T Y / Q)$ & $q(r q / \cdot)$ & دييلم & \\
\hline & $r(q / V)$ & $F(\mid r / q)$ & بالاتر از دييلم & \\
\hline \multirow{2}{*}{$\cdot 19 \cdot V$} & $I V(\Delta F / \Lambda)$ & $19(91 / \pi)$ & كارمند & \multirow{2}{*}{ شغل بدر } \\
\hline & $\mid f(F d / T)$ & $I r(r \wedge / V)$ & آزاد & \\
\hline \multirow{2}{*}{$\cdot / 1919$} & $V(T Y / G)$ & $F(\mid r / q)$ & كارمند & \multirow{2}{*}{ شغل مادر } \\
\hline & $T F(V V / \mathcal{F})$ & $r V(\Lambda V / I)$ & خانه دار & \\
\hline \multirow{3}{*}{$\cdot / \cdot v \Delta$} & $q($ ( //•) & $\Delta(\mid \& / 1)$ & خوب & \multirow{3}{*}{ خانواده } \\
\hline & $19(91 / \Gamma)$ & $19(\Delta / / 9)$ & متوسط & \\
\hline & $r(9 / V)$ & $1 \cdot\left(T_{T / K}\right)$ & ضعيف & \\
\hline
\end{tabular}


مستقل انجام شد و نتايج به دست آمده نشان دهنده اين بود

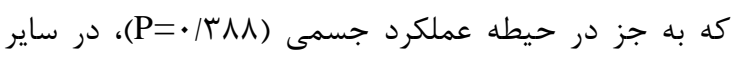
حيطه ها و نيز نمره كل كيفيت زندگى ديط تفاوت آمارى

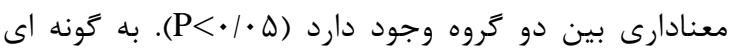

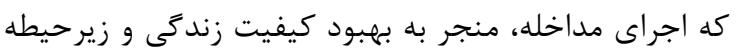

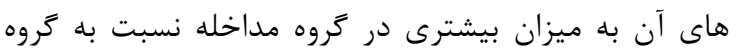

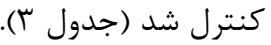

بود كه به جز در حيطه محدوديت عملكرد جسمى

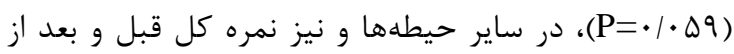

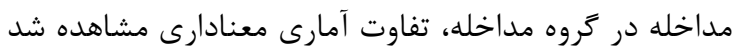

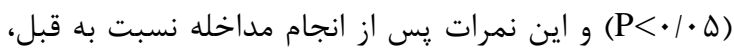

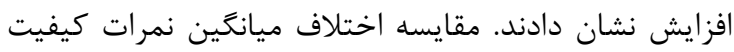

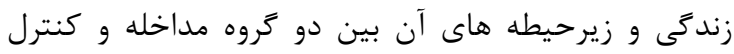

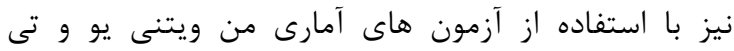

جدول بّ: مقايسه اختلاف ميانكَين و انحراف معيار نمرات كيفيت زندگى و زيرحيطه هاى آن به تفكيك دو كروه مداخله وكنترل

\begin{tabular}{|c|c|c|c|c|c|c|c|}
\hline \multirow{2}{*}{ p-value ** } & \multicolumn{3}{|c|}{ كروه كنترل } & \multicolumn{3}{|c|}{ كروه مداخله } & \multirow{2}{*}{ حيطه ها } \\
\hline & p-value* & بعد از مداخله & قبل از مداخله & p-value* & بعد از مداخله & قبل از مداخله & \\
\hline$\cdot / r \wedge \Lambda$ & • & $V V / T \Delta \pm I N / \Delta T$ & $V N / \Delta F \pm r \cdot \mid \Lambda \varphi$ & .1 .19 & $\Lambda F / r \Delta \pm I V / \Delta F$ & $\Lambda \cdot / r T \pm 19 / \mathbb{R}$. & عملكرد جسمى \\
\hline$\cdot / \cdot r$ & .1 .19 & $\Delta \Delta / \varepsilon \mathcal{F} \pm f \mid / \Lambda \Lambda$ & $q T / V \cdot \pm r \Delta / r q$ & $\cdot 1 \cdot 09$ & $\wedge r / \cdot \varepsilon \pm r \varepsilon / q 1$ & $V \Delta / \cdot \cdots \pm r V / \cdot \wedge$ & جسمى محدويت عملكرد \\
\hline $.1 \cdot f$. & . MFT & $\Delta \Lambda / \cdot G \pm F \mid / r q$ & $G \Psi / F F \pm r F / \Lambda$. & .1 .19 & $V \Delta / T \varphi \pm \mu r /{ }^{\prime} q$ & $q K / F F \pm F \cdot 19 q$ & عاطفدى محيت نقش \\
\hline$\cdot / \cdot r q$ & . IGYA & $\varepsilon r / \Delta \Lambda \pm 19 / 9 \Delta$ & $q 1 / 4 \Delta \pm r \cdot / \cdot q$ & $\cdot / \cdot \cdot 1$ & $V(/ 9 r \pm 19 / \cdot F$ & $G F / \cdot r \pm r r / \Delta r$ & شادابى و نشاط \\
\hline$<\cdot / \cdot \cdot 1$ & $\cdot 1 \cdot 19$ & $\Delta N / \cdot G \pm r r / q 9$ & $q r / \cdot q \pm r r / l F$ & $\cdot 1 \cdot \cdot 1$ & $V T / T \Delta \pm|N / F|$ & $s r / r \Delta \pm r V / \cdot r$ & سلامت روان \\
\hline$<\cdot / \cdot \cdot 1$ &.$|9| f$ & $V G / I T \pm r r / I F$ & $V N / \cdot r \pm|N / q|$ & $\cdot / \cdot r$ & $\Lambda \Delta / G r \pm r \cdot / r \Delta$ & $V T / \cdot \varphi \pm r \cdot / 1 \Lambda$ & عملكرد اجتماعى \\
\hline$\cdot / \cdot r$ & $\cdot / 491$ & $V I / r \Lambda \pm r r / \cdot V$ & $V F / A T \pm Y Y / D I$ & $\cdot / \cdot \wedge$ & $\Lambda I / \Lambda \Lambda \pm r r / \Delta r$ & $V r / V q \pm r \Delta / \varphi \Delta$ & درد بدنى \\
\hline$<\cdot / \cdot \cdot 1$ & $\cdot / \cdot V r$ & $\Delta T / T r \pm T \& / \cdot \Delta$ & $\Delta q / r r \pm r r / \& q$ & $<\cdot / \cdot \cdot 1$ & $V r / r q \pm r / / R$ & $\Delta q / F) \pm r r / q r$ & سلامت عمومى \\
\hline$<\cdot / \cdot \cdot 1$ & . / & $q r / q r \pm r \cdot N \cdot$ & $q V / \Lambda \cdot \pm 19 / T^{\prime}$ & $<\cdot 1 \cdot \cdot \cdot 1$ & $V \Lambda / T Y \pm \mid \varepsilon / \Delta V$ & $9 N / 9 r \pm r I / 9 \Delta$ & نمره كل \\
\hline
\end{tabular}

همكاران نشانكر اين بود كه كيفيت زندكى كودكان مبتلا به

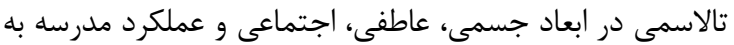

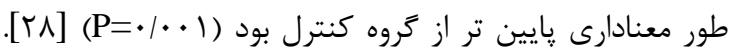

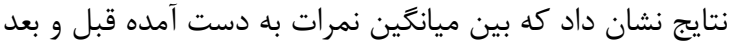

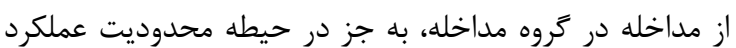
جسمى، در ساير حيطه هاى كيفيت زندگى تفاوت هاى آمارى

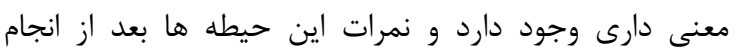

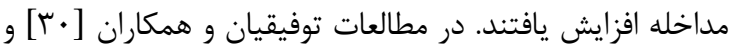

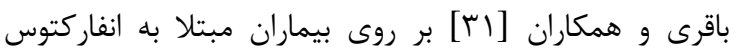

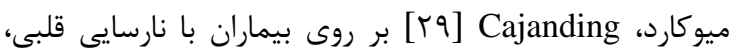

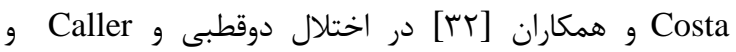

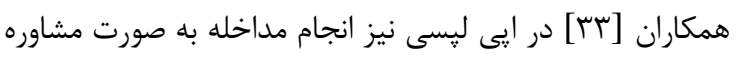

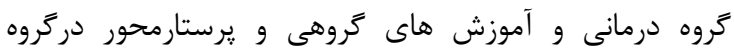
مداخله باعث افزايش قابل ملاحظه در نمرات ميانگين هريك از ازئ

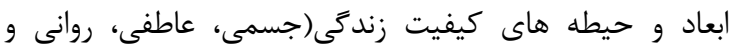

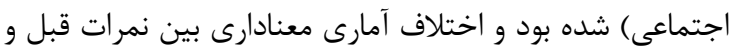
بعد از اجراى مداخله وجود داشت. همجنين درد درد مطالعات انجام

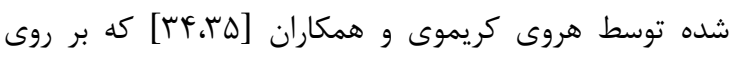

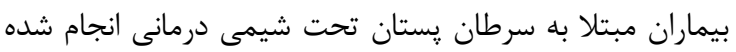

تالاسمى مازور، شايع ترين بيمارى ارثى در دنيا و كشور

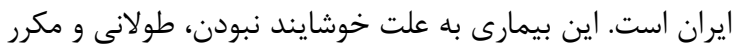

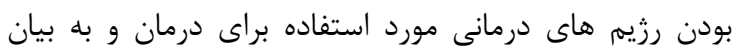

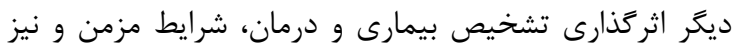

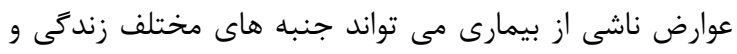

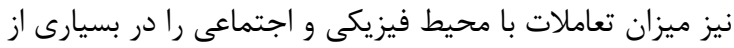

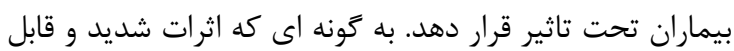

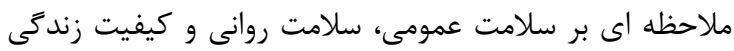

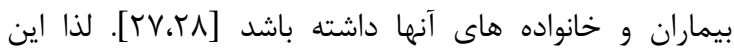
مطالعه با هدف تعيين تاثير مشاوره بر كيفيت زندكى بيمارن انداران

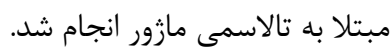

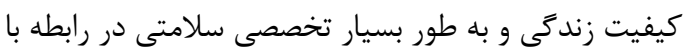
كيفيت زندكى حيطه هاى سلامت جسمى، روانى و اجتماعى را

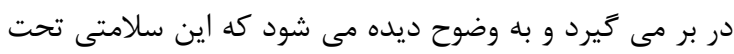

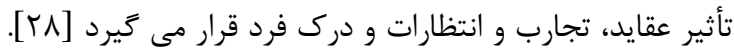

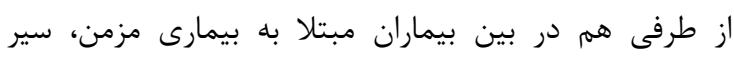

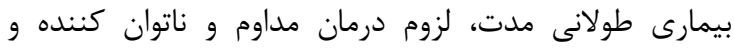

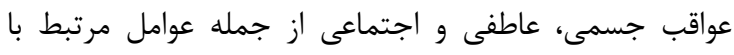

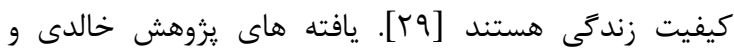


استرس زاى محيطى بر نقش يذيرى اجتماعى تاثير بخذارد.

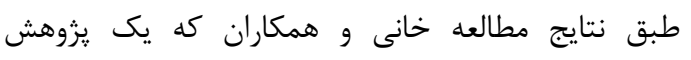

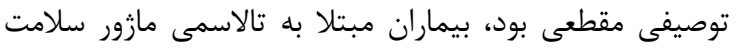

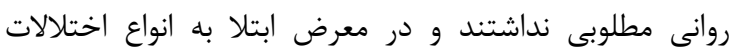

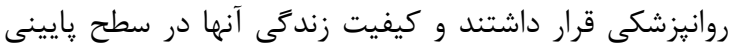

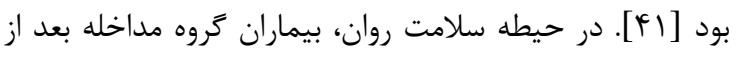

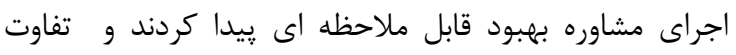

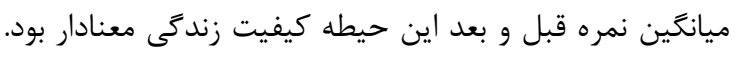

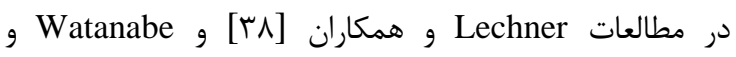

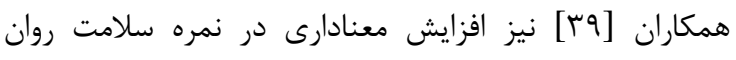

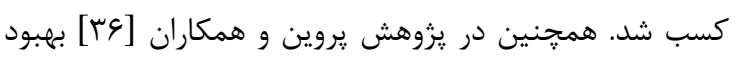

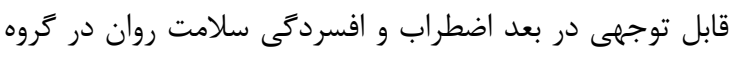

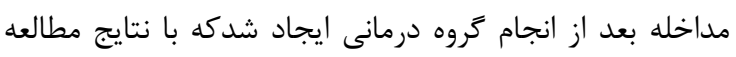

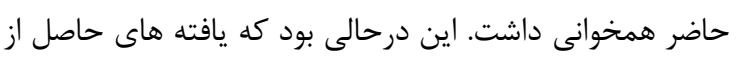

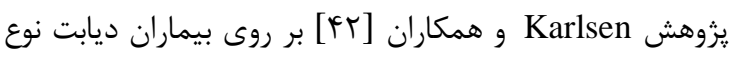

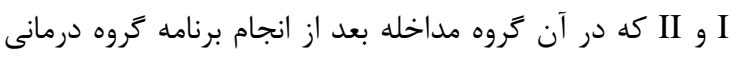

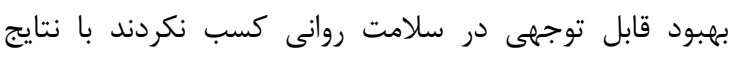

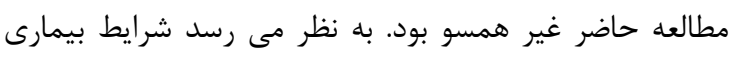

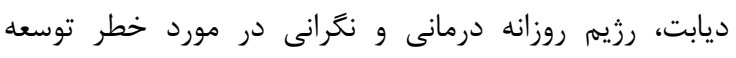

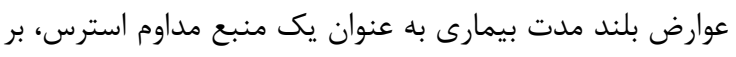
سلامت روان بيماران اثركذار بوده است.

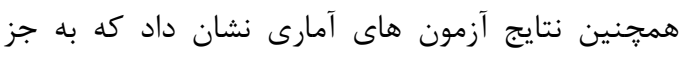

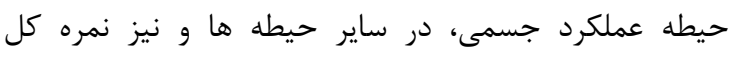

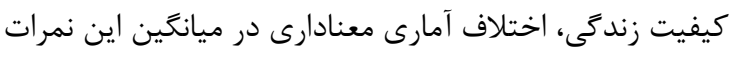

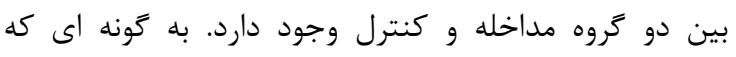

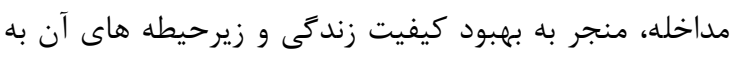

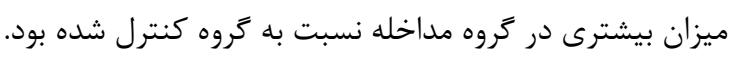

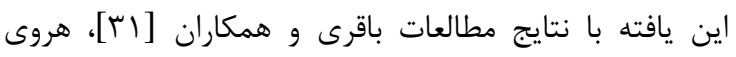

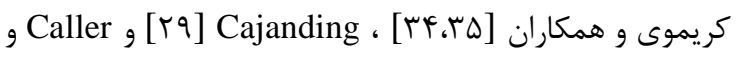

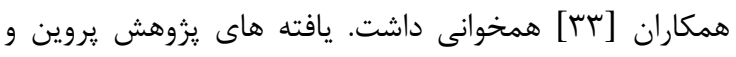

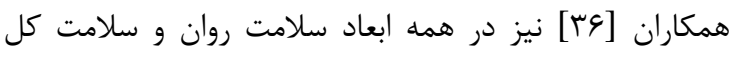

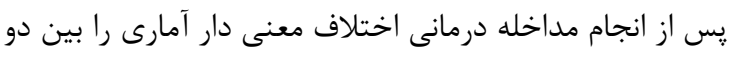

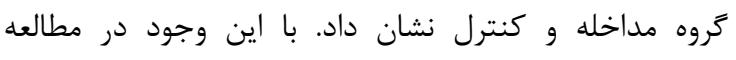

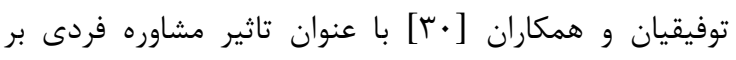

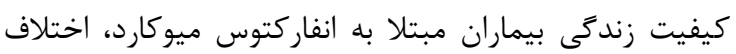

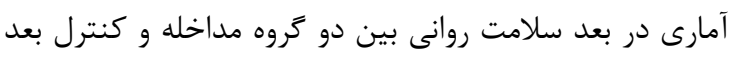

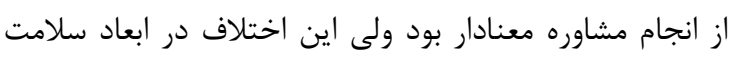

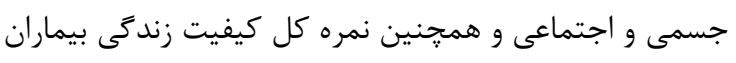

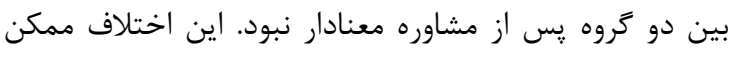

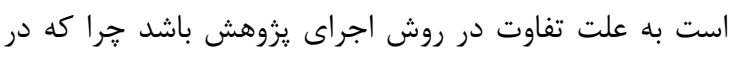

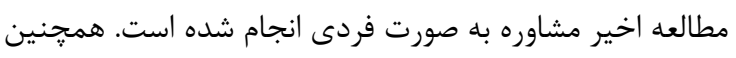

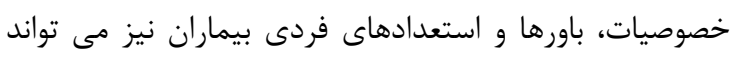
در اين نتيجه دخيل باشد. در اجراى اين يزوهش محدوديتهايى در زمينه همكارى
بود، اجراى مشاوره باعث بهبود قابل توجه در نمرات مقياس هاى إى

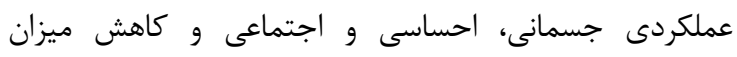

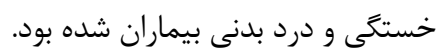

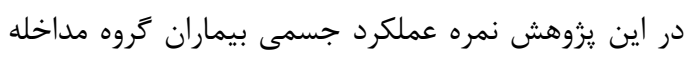

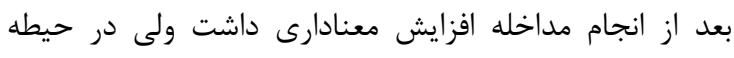

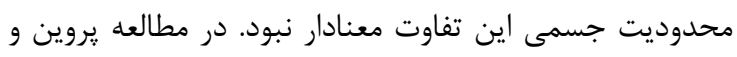

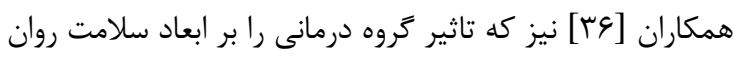

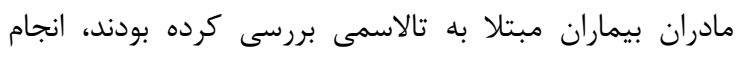

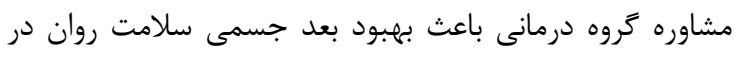

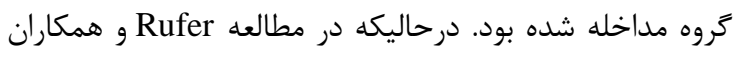

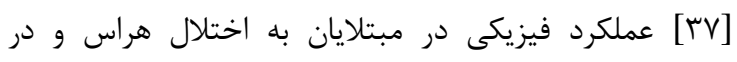

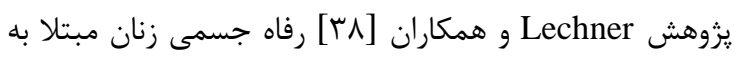

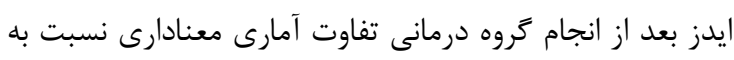

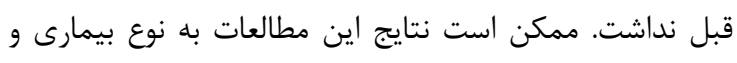

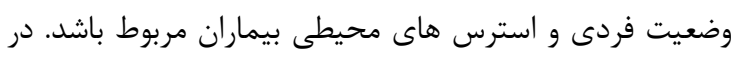

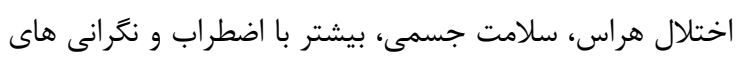

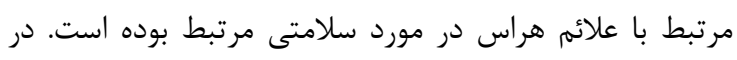

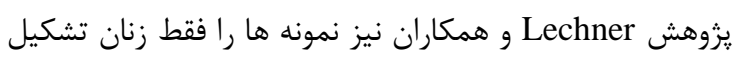

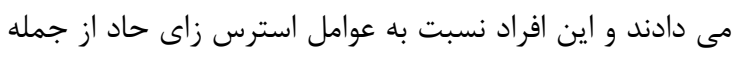

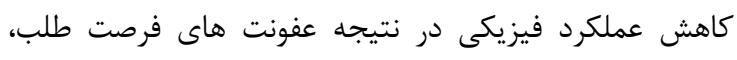

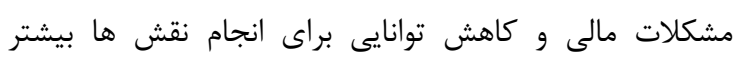
مستعد و آسيب يذير هستند.

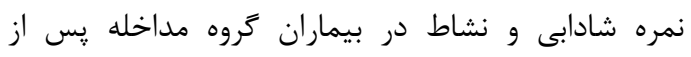

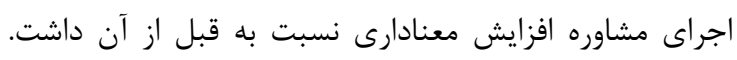

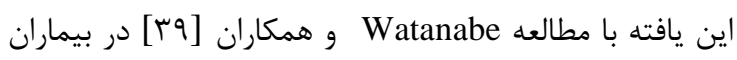

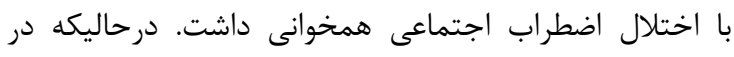

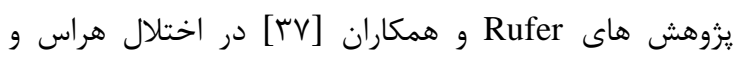

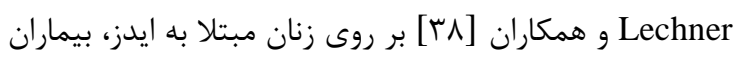

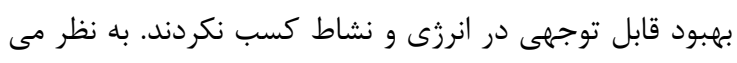

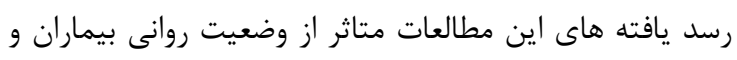

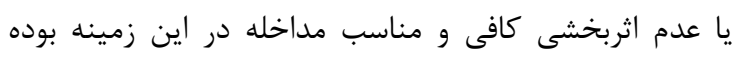

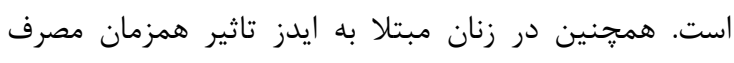

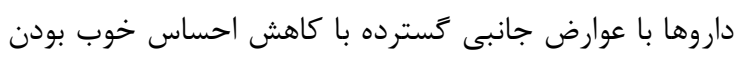

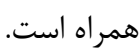
نمره عملكرد اجتماعى بيماران در كروه مداخله بعد از انجام

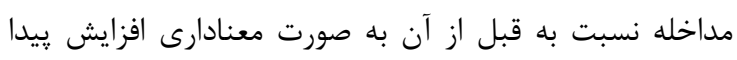

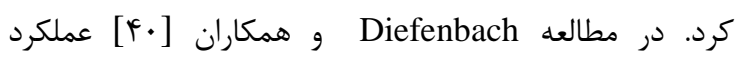

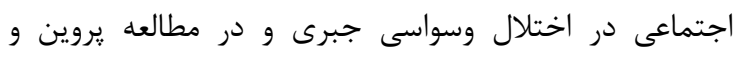

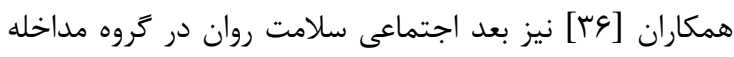

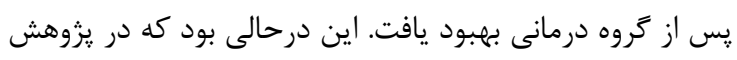
Lechner

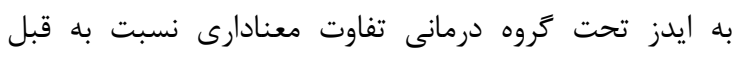

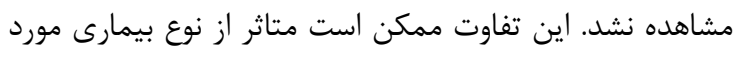

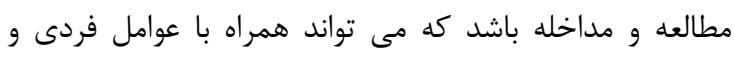




\section{تشكر و قلرواذى}

اين مقاله از پايان نامه كارشناسى ارشد دانشكده يرستارى و و

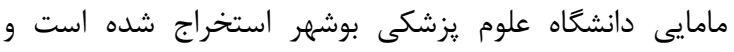

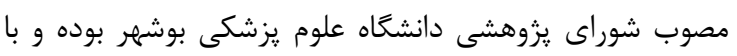

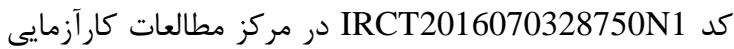
بالينى ايران ثبت شده است. از مركز توسعه يزوهش هاى اين

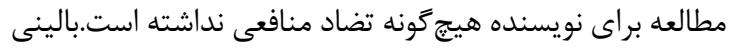
بيمارستان شهداى خليج فارس بوشهر، مسئولين محترم مركز

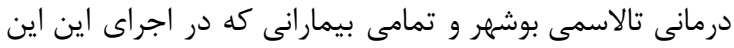

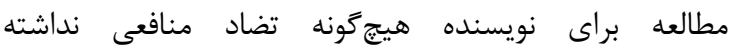
است. يزوهش ما را يارى نمودند، سياسگز اريم.

تضاد منافع اين مطالعه براى نويسنده هيجَّونه تضاد منافعى نداشته

\section{REFERENCES}

1. Shasti S, Mirhaghjou S N, Masouleh Sh R, Emami Sigarodi A, Atrkar Roshan Z. Study the Life Skills of 11-19 year old Children affected by Thalassemia referring to Educational and Remedial Centers in Rasht city from their Mothers' Point of View 2009-2010. Journal of School of Nursing and Midwifery, Guilan. 2010; 20(63):16 - 21 (Persian)

2. Galanello R, Origa R. Beta-thalassemia.Orphanet. Journal of Rare Diseases. 2010; 5(11):1-15

3. El Dakhakhny A M, Hesham M A, Mohamed S E, Mohammad F N. Quality of Life of School Age Thalassemic Children at Zagazig City. Journal of American Science. 2011;7(1):186 - 97

4. Saki N, Dehghanifard A, Kaviani S, Jalali Far M A, Mousavi S H, Al Ali K,et al. Beta Thalassemia: Epidemiology and Diagnostic and Treatment Approaches in Iran. Genetics in the Third Millennium. 2012; 10(1):267483 (Persian)

5. Khodaei Gh H, Farbod N, Zarif B, Nateghi S, Saeidi M. Frequency of thalassemia in Iran and Khorasan Razavi. International journal of Pediatrics. 2013; 1(1): 45-50. (Persian)

6. Zarea K, Baraz Pordanjani Sh, Pedram M, Pakbaz Z. Quality of Life in Children with Thalassemia who Referred to Thalassemia Center of Shafa Hospital. Care of chronic diseases Jundishapur J. 2012; 1(1):45-53. (Persian)

7. Surapolchai $\mathrm{P}$, Satayasai $\mathrm{W}$, Sinlapamongkolkul $\mathrm{Ph}$, Udomsubpayakul U. Biopsychosocial Predictors of HealthRelated Quality of Life in Children with Thalassemia in Thammasat University Hospital. J Med Assoc Thai. 2010; 93(7): 65-75

8. Yazdi Kh, Sanagou A, Jouybari L. Psychosocial, social and financial problems with coping strategies among families with thalassemic patients. Journal of Gorgan University of Medical Sciences .2007; 9(2):71-5. (Persian)

9. Siddiqui S H, Ishtiaq R, Sajid F, Sajid R. Quality of Life in Patients with Thalassemia Major in a Developing Country. Journal of the College of Physicians and Surgeons Pakistan. 2014; 24(7):477- 80.

10. Caocci G, Efficace F, Ciotti F, Roncarolo M G,Vacca A, Piras E,et al. Health related quality of life in Middle Eastern children with beta-thalassemia. BMC Blood Disorders 2012; 12(6):1-7

11. Alijany-Renany H, Tamaddoni A, Haghighy-zadeh M H, Pourhosein S. The effect of using partnership care model on
برخى بيماران در شركت در جلسات مشاوره و همجنين تعداد

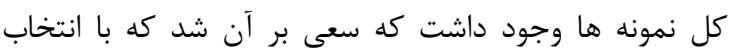

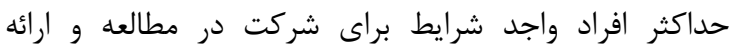
توضيحات لازم در خصوص هدف انجام يزوهش و تاثيركذارى آن بر بيماران، اين محدوديت ها كاهش يابند.

\section{نتيجه تيرى}

نتايج يزوهش حاضر نشان داد كه اجراى برنامه درمانى

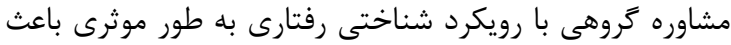

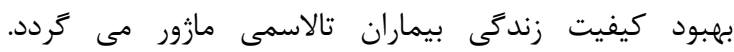

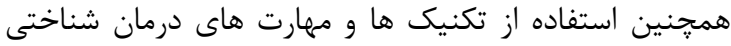

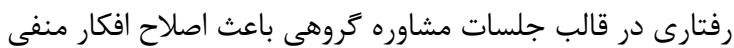

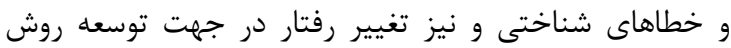

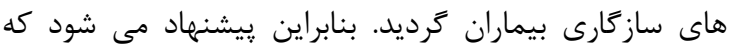

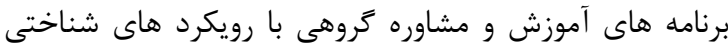

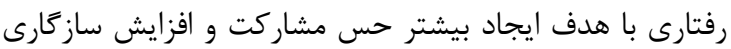

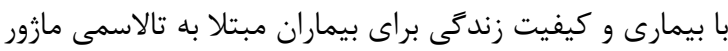

the quality of life in the school-age children with $\beta$ thalassemia. Journal of Shahrekord University of Medical Sciences. 2012; 14(1): 41- 9. (Persian)

12. Ismail A, Campbell M J, Ibrahim H M, Jones G L. Health related quality of life in Malaysian children with thalassemia. Health and Quality of Life Outcomes. 2006; 4(39): $1-8$

13. Baghianimoghadam $M H$, Sharifirad $G$ h, Rahaei Z, Baghianimoghadam B, Heshmati. Health Related Quality of Life in children with Thalassemia assessed on the basis of SF-20 Questionnaire in Yazd, Iran: A Case-Control Study. Central European journal of public health. 2011; 19(3): 16569. (Persian)

14. Zeighami Mohammadi Sh, Tajvidi M. Relationship between spiritual well-being with hopelessness and social skills in Beta-thalassemia major adolescents (2010). Modern Care Journal. 2011; 8(3):116 - 24. (Persian)

15. Salehi M, Mirbehbahani N B, Jahazi A, Aghebati Amlashi Y. General health of beta-thalassemia major patients in Gorgan , Iran. Journal of Gorgan University of Medical Sciences. 2014; 16 (1):120- 5. (Persian)

16. Hajibeigi B, Azarkeyvan A, Alavian S M, Moghani Lankarani M, Assari Sh. Anxiety and depression affects life and sleep quality in adults with beta-thalassemia. Indian Journal of Hematology and Blood Transfusion. 2009; 25(2): 59- 65. (Persian)

17. Mazzone L, Battaglia L, Andreozzi F, Romeo M A, Mazzone D. Emotional impact in $\beta$-thalassemia major children following cognitive - behavioural family therapy and quality of life of caregiving Mothers. Clinical Practice and Epidemiology in Mental Health. 2009; 5(5):1- 6

18. Anie KA, Massaglia P. Psychological therapies for thalassemia (Review). The Cochrane Library. 2014; (3):1-12

19. Ramezani T. Depression and counselling needs in women with breast cancer reffering to chemotherapy in Kerman. Andeesheh Va Raftar. 2000; 6(4):70 -7 (Persian)

20. Chesson R A, Chisholm D, Zaw W. Counseling children with chronic physical illness. Patient Education and Counseling. 2004;55(3):331-8

21. Kheirkhah M, Vahedi M, Jenani S P. The Effect of Group Counseling on Infertility Adjustment of infertile women in Tabriz Al-Zahra clinic. The Iranian Journal of Obstetrics, Gynecology and Infertility. 2014; 17(113):7-14. (Persian)

22. Modarres M, Rahimikian F, Mehran A. Impact of PreHysterectomy Counseling on Depression among Patients 
Reffered to TUMS Hospitals. Hayat, Journal of hayat. 2013; 19(2): 40-50. (Persian)

23. Hadi N, karami D, Montazeri A. Health-related quality of life in patients with thalassemia major .Payesh quarterly. 2009; 8(4): 387-93. (Persian)

24. Qu B, Guo HQ, Liu J, Zhang Y, Sun G. Reliability and validity testing of the SF-36 questionnaire for the evaluation of the quality of life of Chinese urban construction workers. Journal of International Medical Research. 2009; 37(4):1184- 90

25. Ware J E, Gandek B. Overview of the SF-36 Health Survey and the International Quality of Life Assessment (IQOLA) Project. Journal of Clinical Epidemiology. 1998; 51(11): 903-12

26. Montazeri A, Goshtasebi A, Vahdaninia M. Translation, reliability and validity of the Persian version of the SF-36 standard tool. Journal of Payesh. 2006; 5(1): 49-56. (Persian)

27. Jafari H, Lahsaeizadeh S, Jafari P, Karimi M. Quality of life in thalassemia major: Reliability and validity of the Persian version of the SF-36 questionnaire. Journal of postgraduate medicine. 2008; 54(4):273-5. (Persian)

28. Khaledi Sh, Moridi G, Valiee S. Comparison the quality of life of healthy and Thalassemic children.Nursing Research. 2013;8(29):87-94. (Persian)

29. Cajanding RJ M. The Effectiveness of a Nurse-Led Cognitive-Behavioral Therapy on the Quality of Life, SelfEsteem and Mood Among Filipino Patients Living With Heart Failure: a Randomized Controlled Trial. Applied Nursing Research. 2016; 31:86-93

30. Tofighiyan T, Najjar L, Akaberi A, Shegarf Nakhaei M R. The effect of individual counseling on quality of life of patients with myocardial infarction .J Sabzevar Univ Med Sci. 2010; 16(4):206-12. (Persian)

31. Bagheri H, Memarian R, Elhani F. The effects of group counseling on quality of life in patients with myocardial infarction admitted to Hospital Clinic of Imam Khomeini and Shariati Tehran. Hakim Research Journal. 2006; 6(4):89- 95. (Persian)

32. Costa RT, Cheniaux E, Rangé B P, Versiani M, Nardi A E. Group cognitive behavior therapy for bipolar disorder can improve the quality of life. Brazilian Journal of Medical and Biological Research. 2012; 45(9): 862- 8

33. Caller TA, Ferguson R J, Roth R M, Secore K L, Alexandre F P, Zhao W,et al. A cognitive behavioral intervention
(HOBSCOTCH) improves quality of life and attention in epilepsy. Epilepsy \& Behavior. 2016; 57:111- 7

34. Heravi Karimoi M, Pourdehghan M, Faghih Zadeh S, Montazeri A, jadid Milani M.The effects of Group Counseling on Symptom Scales of Life Quality in Patients with Breast Cancer Treated by Chemotherapy. J Kermanshah Univ Med Sci. 2006; 10(1):10 -22. (Persian)

35. Heravi Karmoi M, Pourdehghan M, Faghih Zadeh S, Montazeri.The effects of Group Counseling on Functional Scales of Life Quality in Patients with Breast Cancer Treated by Chemotherapy. Scientific Journal of Shahed University. 2006; 13(62):69 -78. (Persian)

36. Parvin N, Alavi A, Alidoust E, Forouzandeh N, Hosein zadeh Sh, Kamkhah A, et al. The effects of group therapy on mental health condition in mothers of thalassemic patients. Journal of Shahrekord Uuniversity of Medical Sciences. 2008;10(3): 37-43. (Persian)

37. Rufer M, Albrecht R, Schmidt O, Zaum J, Schnyder U, Hand I, et al. Changes in quality of life following cognitivebehavioral group therapy for panic disorder. European Psychiatry. 2010; 25(1): 8-14

38. Lechner S C, Antoni M H, Lydston D, LaPerriere A, Ishii $\mathrm{M}$, Devieux J,et al. Cognitive-behavioral interventions improve quality of life in women with AIDS. Journal of Psychosomatic Research. 2003; 54(3): 253- 61

39. Watanabe N, Furukawa TA, Chen J, Kinoshita Y, Nakano $\mathrm{Y}$, Ogawa S,et al. Change in quality of life and their predictors in the long-term follow-up after group cognitive behavioral therapy for social anxiety disorder: a prospective cohort study. BMC psychiatry. 2010;10(1): 1-10

40. Diefenbach G H, Abramowitz J S, Norberg M M, Tolin D $\mathrm{S}$. Changes in quality of life following cognitive-behavioral therapy for obsessive-compulsive disorder. Behaviour Research and Therapy. 2007; 45(12):3060- 8

41. Khani H, Majdi M R, Azad Marzabadi E, Montazeri A,Ghorbani A, Ramezani M,et al. Quality of life in Iranian Beta-thalassemia major patients of southern coastwise of the Caspian Sea. Journal of Behavioral Sciences. 2009; 2(4):325 -32. (Persian)

42. Karlsen B, Idsoe Th, Dirdal I, Hanestad B R, Bru E. Effects of a group-based counselling program on diabetesrelated stress, coping, psychological well-being and metabolic control in adults with type 1 or type 2 diabetes. Patient Education and Counseling. 2004; 53(3): 299 - 308 